\title{
Tracking Control of a Ship by PI-Type Sliding Controller
}

Ker-Wei Yu

Department of Marine Engineering, National Kaohsiung Marine University, 142, Hai-Chuan Rd. Nan-Tzu, Kaohsiung, Taiwan 811., kwyu@mail.nkmu.edu.tw

Chen-En Wu

Department of Marine Engineering, National Kaohsiung Marine University, 142, Hai-Chuan Rd. Nan-Tzu, Kaohsiung, Taiwan 811.

Follow this and additional works at: https://jmstt.ntou.edu.tw/journal

Part of the Engineering Commons

\section{Recommended Citation}

Yu, Ker-Wei and Wu, Chen-En (2004) "Tracking Control of a Ship by PI-Type Sliding Controller," Journal of Marine Science and Technology. Vol. 12: Iss. 3, Article 7.

DOI: $10.51400 / 2709-6998.2237$

Available at: https://jmstt.ntou.edu.tw/journal/vol12/iss3/7

This Research Article is brought to you for free and open access by Journal of Marine Science and Technology. It has been accepted for inclusion in Journal of Marine Science and Technology by an authorized editor of Journal of Marine Science and Technology. 


\section{Tracking Control of a Ship by PI-Type Sliding Controller}

Acknowledgements

This work was supported by the National Science Council, Taiwan, Republic of China, under Grant NSC 92-2611-E-022-005.

This research article is available in Journal of Marine Science and Technology: https://jmstt.ntou.edu.tw/journal/ 


\title{
TRACKING CONTROL OF A SHIP BY PI-TYPE SLIDING CONTROLLER
}

\author{
Ker-Wei Yu* and Chen-En Wu
}

Key words: PI controller, variable structure control, sliding mode

\begin{abstract}
In this paper, the tracking control of ship maneuvering by a modified PI-type sliding controller is investigated. This scheme is composed of three components. The first component is the equivalent control, which is designed to assure that the state trajectory of the system remains in the sliding surface and the nominal system is stable in the absence of system uncertainties and disturbance. The second and third components are proportional-type and integral-type variable structure controllers, respectively. Several numerical examples of ship handling, namely course keeping, course changing, and course tracking are given to demonstrate the applicability of the proposed control scheme.
\end{abstract}

\section{INTRODUCTION}

As is well known, ship maneuvering is critical for marine safety. In the past, several design methods, e.g., classical PID control, adaptive control [1], and other modern control technique $[6,8,9,12,13,16,17]$, for autopilots have been proposed. The maneuvering problems including course keeping, course tracking, berthing, and anti-collision were considered in those papers. As the nonlinear behavior involved in ship maneuvering is very complicated, characterization of ship maneuvering behavior by a pure mathematical model is usually difficult. In real case of ship maneuvering, the parameter uncertainties and external disturbance should be considered. Consequently, the robustness of controlled system is one of the important factors in controller design.

In recent years, "robust control" is one of the most popular topics in control field. Many researchers have devoted themselves into this subject by utilizing different design methodology. One of the famous methods is

Paper Submitted 04/19/04, Accepted 08/27/04. Author for Correspondence: Ker-Wei Yu.E-mail: kwyu@mail.nkmu.edu.tw.

*Department of Marine Engineering, National Kaohsiung Marine University, 142, Hai-Chuan Rd. Nan-Tzu, Kaohsiung, Taiwan 811. the so-called variable structure control (VSC) [14] with sliding mode strategy. It has been proven as an effective and robust control technology and also has been applied in many practical applications [2, 3, 5, 7, 10, 11, 15].

Recently, Chern and Wu proposed a control scheme called integral variable structure control (IVSC) [4] in which an integral controller was added to a variable structure controller, so that not only the uncertainties, parameter variations and/or disturbances can be eliminated, but also resulted in zero steady-state error under step input. Fung and other co-researchers [7], based on the integral variable structure control scheme, developed a switching control law between proportional-type sliding mode controller and integral-type sliding mode controller. The compensating PI controller improved design method of IVSC, not only it inherits all the advantages of IVSC, but also it eliminates most of the major limitations of IVSC. However, the stability cannot be guaranteed within switching controller motion. The requirements of system performance just mentioned are very important in ship maneuvering. Large overshoot and steady-state error are not allowed in course tracking to desired path or navigation in narrow channel. The main purpose of this study is to construct a PI-type sliding controller to obtain fast converging motion with little overshoot.

This paper is organized as follows. Section 2 describes the construction of a PI-type sliding controller for a class of nonlinear systems. In section 3 , three illustrative examples of ship maneuvering are provided to illustrate the use of the proposed control scheme. Finally, brief conclusions are drawn in section 4.

\section{COMPENSATONG PIVSC}

Consider a dynamic system described as:

$$
\begin{aligned}
& \dot{X}_{i}=X_{i+1}, \quad i=1,2, \ldots, n-1, \\
& \dot{X}_{n}=-\sum_{i=1}^{n} a_{i} X_{i}+b U-f(t), \\
& Y=X_{1} .
\end{aligned}
$$


Here $X=\left[\begin{array}{lll}X_{1} & \ldots & X_{n}\end{array}\right]^{T}$ is the state vector, $Y$ is the controlled output, $a_{i}$ and $b$ are constant or time-varying system parameters, and $f(t)$ is the disturbance. Let

$$
a_{i}=a_{i}^{0}+\Delta a_{i}, \quad b=b^{0}+\Delta b, i=1,2, \ldots, n,
$$

where $a_{i}^{0}, b^{0}$ are nominal values, and $\Delta a_{i}, \Delta b$ are the variations of $a_{i}$ and $b$. Without loss of generality, we assume that $b^{0}>0$ and $b^{0}+\Delta b>0$. Furthermore, assume

$$
|f(t)| \leq F(t),|\Delta b| \leq N_{b},\left|\Delta a_{i}\right| \leq N_{a i}, i=1, \ldots, n,
$$

where $F(t) \geq 0, N_{b} \geq 0, N_{a i} \geq 0$ are the maximum upper bounds of allowed uncertainties, respectively.

The control function $U$ depends on the switching function $\sigma$ given by

$$
\sigma=-K_{P} r-w_{1} K_{I} Z+\sum_{i=1}^{n} c_{i} X_{i}
$$

In the defining equation for the switching function, $r$ is the desired tracking command, $X_{1}-r$ is the tracking error, $Z$ is the integral of the tracking error satisfying $Z$ $=r-X_{1}, K_{P}$ and $K_{I}$ are defined as the gains of proportional and integral controllers, respectively, and $c_{i}, i=$ $1,2, \ldots, n$, with $c_{1}=K_{P}+w_{1}$ and $c_{n}=1$, are design parameters. From (1), we have

$$
\begin{aligned}
\dot{\sigma}= & -\sum_{i=1}^{n} a_{i} X_{i}+\sum_{i=2}^{n} c_{i-1} X_{i}-K_{P} g-w_{1} K_{I}\left(r-X_{1}\right) \\
& +b U-f(t),
\end{aligned}
$$

where $g=\dot{r}$.

Let the control function $\mathrm{U}$ be decomposed as

$$
U=U^{0}+U_{s w P}+U_{s w I}
$$

where $U^{0}$, called the equivalent control, is defined as the solution of the problem $\dot{\sigma}=0$ under $\Delta a_{i}=0, \Delta b=0$, and $f(t)=0$. That is

$$
U^{0}=\frac{\left(\sum_{i=1}^{n} a_{i}^{0} X_{i}-\sum_{i=2}^{n} c_{i-1} X_{i}\right)+K_{P} g+w_{1} K_{I}\left(r-X_{1}\right)}{b^{0}} .
$$

Under the same conditions, when $U^{0}$ is substituted into (1), the "nominal system" is guaranteed to be stable if we choose the parameters $c_{1}, \ldots, c_{n-1}, w_{1}$, and $K_{I}$ such that the following polynomial is Hurwitz:

$$
s^{n}+c_{n-1} s^{n-1}+\ldots+c_{1} s+w_{1} K_{I}
$$

The components $U_{s w P}$, the proportional-type control, and $U_{s w I}$, the integral-type control, are used to eliminate the influence due to the plant parameter variation in $\Delta a_{i}, \Delta b$, and disturbance $f(t)$ in order to guarantee the existence of a sliding control. They are given by

$$
U_{s w P}=\phi_{1 P}\left(X_{1}-r\right)+\sum_{i=2}^{n} \phi_{i P} X_{i}
$$

and

$$
U_{s w I}=\phi_{1 I}\left(X_{1}-K_{I} Z\right)+\sum_{i=2}^{n} \phi_{i I} X_{i}+\phi_{n o}
$$

where

$$
\phi_{1 P}= \begin{cases}\rho_{1 P}, & \text { if }\left(X_{1}-r\right) \sigma>0 \\ \varsigma_{1 P}, & \text { if }\left(X_{1}-r\right) \sigma<0\end{cases}
$$

$\phi_{i P}=\left\{\begin{array}{ll}\rho_{i P}, & \text { if } X_{i} \sigma>0, \\ \varsigma_{i P}, & \text { if } X_{i} \sigma<0,\end{array} \quad i=2, \ldots, n\right.$,

$\phi_{1 I}= \begin{cases}\rho_{1 I}, & \text { if }\left(X_{1}-K_{I} Z\right) \sigma>0, \\ \varsigma_{1 I}, & \text { if }\left(X_{1}-K_{I} Z\right) \sigma<0,\end{cases}$

$$
\phi_{i I}=\left\{\begin{array}{ll}
\rho_{i I}, & \text { if } X_{i} \sigma>0, \\
\varsigma_{i I}, & \text { if } X_{i} \sigma<0,
\end{array} \quad i=2, \ldots, n,\right.
$$

and

$$
\phi_{n o}=\left\{\begin{array}{lll}
\rho_{n o}, & \text { if } & \sigma>0 \\
\varsigma_{n o}, & \text { if } & \sigma<0
\end{array}\right.
$$

For the existence of a sliding motion of the closedloop trajectories on the switching surface $\sigma=0$, we must have [11]

$$
\lim _{\sigma \rightarrow 0} \sigma \dot{\sigma}<0
$$

Substituting equations (7) and (9) into equation (5) leads to

$$
\begin{aligned}
\dot{\sigma}= & -\sum_{i=1}^{n} \Delta a_{i} X_{i}-f(t) \\
& +B\left(\sum_{i=1}^{n} a_{i}^{0} X_{i}-\sum_{i=2}^{n} c_{i-1} X_{i}+K_{P} g+w_{1} K_{I}\left(r-X_{1}\right)\right) \\
& +b\left(\phi_{1 P}\left(X_{1}-r\right)+\sum_{i=2}^{n} \phi_{i P} X_{i}\right) \\
& +b\left(\phi_{1 I}\left(X_{1}-K_{I} Z\right)+\sum_{i=2}^{n} \phi_{i I} X_{i}+\phi_{n o}\right)
\end{aligned}
$$

where $B \equiv \Delta b / b^{0}$. Then we have 


$$
\begin{aligned}
& \sigma \dot{\sigma} \leq\left\{\left(\left(\frac{N_{a 1}}{2}+\left|\frac{Q a_{1}^{0}}{2}\right|+\left|Q w_{1} K_{I}\right|\right) \operatorname{sign}\left[\left(X_{1}-r\right) \sigma\right]\right.\right. \\
& \left.+\left(b^{0}+N_{b}\right) \phi_{1 P}\right)\left(X_{1}-r\right) \sigma \\
& +\left(\left(\frac{N_{a 1}}{2}+\left|\frac{Q a_{1}^{0}}{2}\right|\right) \operatorname{sign}\left[\left(X_{1}-K_{I} Z\right) \sigma\right]\right. \\
& \left.+\left(b^{0}+N_{b}\right) \phi_{1 I}\right)\left(X_{1}-K_{I} Z\right) \sigma \\
& +\sum_{i=2}^{n}\left(\left(N_{a i}+\left|Q a_{i}^{0}\right|+\left|Q c_{i-1}\right|\right) \operatorname{sign}\left(X_{i} \sigma\right)\right. \\
& \left.+\left(b^{0}+N_{b}\right) \phi_{i P}+\left(b^{0}+N_{b}\right) \phi_{i I}\right) X_{i} \sigma \\
& +\left(\left(F(t)+\left|Q K_{P} g\right|+\left|\frac{N_{a 1} r}{2}\right|+\left|\frac{Q a_{1}^{0} r}{2}\right|\right.\right. \\
& \left.+\left|\frac{N_{a 1} K_{I} Z}{2}\right|+\left|\frac{Q a_{1}^{0} K_{I} Z}{2}\right|\right)
\end{aligned}
$$$$
\left.\left.\left.\cdot \operatorname{sign}(\sigma)+\left(b^{0}+\left|N_{b}\right|\right) \phi_{n o}\right) \sigma\right)\right\},
$$

where $Q \equiv N_{b} / b^{0}$. To satisfy (12), we let

$$
\begin{aligned}
\phi_{1 P} & = \begin{cases}\varsigma_{1 P}, & \text { if }\left(X_{1}-r\right) \sigma<0, \\
-\varsigma_{1 P}, & \text { if }\left(X_{1}-r\right) \sigma>0,\end{cases} \\
\varsigma_{1 P} & =\frac{N_{a 1}+\left|Q a_{1}^{0}\right|+2\left|Q w_{1} K_{I}\right|+2 \varepsilon}{2\left(b^{0}+N_{b}\right)}, \\
\phi_{i P} & = \begin{cases}\varsigma_{i P}, & \text { if } X_{i} \sigma<0, \\
-\varsigma_{i P}, & \text { if } X_{i} \sigma>0,\end{cases} \\
\varsigma_{i P} & =\frac{N_{a i}+\left|Q a_{i}^{0}\right|+\left|Q c_{i-1}\right|+\varepsilon}{2\left(b^{0}+N_{b}\right)}, i=2, \ldots, n,(15 \mathrm{~b}) \\
\phi_{1 I} & = \begin{cases}\varsigma_{11}, & \text { if }\left(X_{1}-K_{I} Z\right) \sigma<0, \\
-\varsigma_{1 I}, & \text { if }\left(X_{1}-K_{I} Z\right) \sigma>0,\end{cases}
\end{aligned}
$$

$$
\begin{aligned}
\varsigma_{1 I} & =\frac{N_{a 1}+\left|Q a_{1}^{0}\right|+2 \varepsilon}{2\left(b^{0}+N_{b}\right)}, \\
\phi_{i I} & = \begin{cases}\varsigma_{i l}, & \text { if } X_{i} \sigma<0, \\
-\varsigma_{i l}, & \text { if } X_{i} \sigma>0,\end{cases} \\
S_{i I} & =\frac{N_{a i}+\left|Q a_{i}^{0}\right|+\left|Q c_{i-1}\right|+\varepsilon}{2\left(b^{0}+N_{b}\right)}, i=2, \ldots, n,
\end{aligned}
$$$$
\phi_{n o}= \begin{cases}\varsigma_{n o}, & \text { if } \sigma<0 \\ -\varsigma_{n o}, & \text { if } \sigma>0\end{cases}
$$

$\varsigma_{n o}=\frac{2 F(t)+2\left|Q K_{P} g\right|+N_{a 1}|r|+\left|Q a_{1}^{0} r\right|+N_{a 1}\left|K_{I} Z\right|+\left|Q a_{1}^{0} K_{I} Z\right|+2 \varepsilon}{2\left(b^{0}+N_{b}\right)}$

where $\varepsilon$ is a small positive number.

\section{ILLUSTRATIVE EXAMPLES}

The nonlinear ship dynamic model [6] relates the yaw angle $\varphi_{r}$ to the rudder angle $\delta_{r}$, as shown in Figure 1 , according to

$$
\ddot{\varphi}_{r}+\frac{K}{T} H\left(\dot{\varphi}_{r}\right)=\frac{K}{T} \delta_{r},
$$

The linearized model of system (17) is shown as follows:

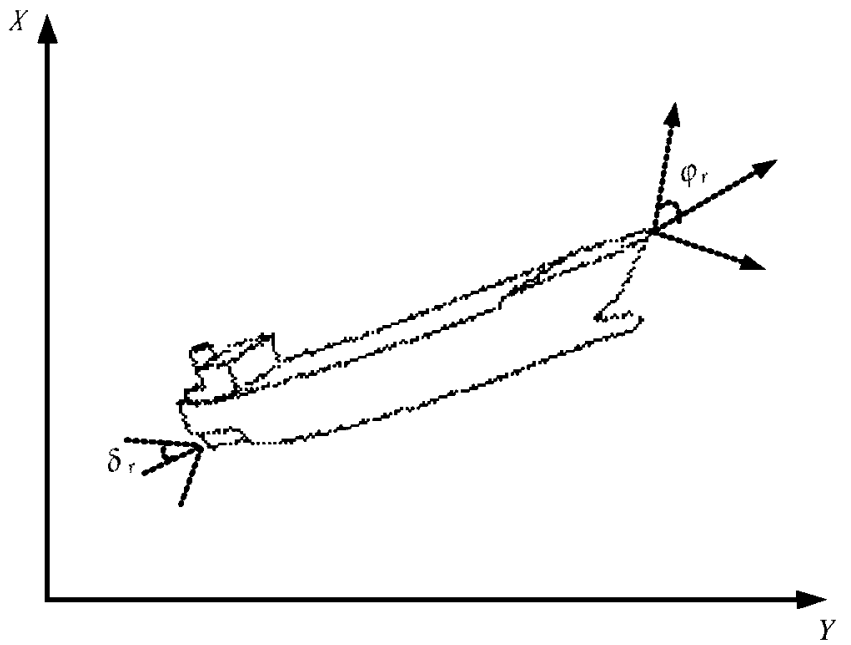

Fig. 1. Motion in the horizontal plane. 


$$
\begin{aligned}
& \dot{x}_{1}=x_{2}, \\
& \dot{x}_{2}=-\frac{K}{T} \alpha x_{2}+\frac{K}{T} \delta_{r},
\end{aligned}
$$

where $K$ is the gain, $T$ is a time constant and $H\left(\dot{\varphi}_{r}\right)=\alpha \dot{\varphi}_{r}$ $+\beta \dot{\varphi}_{r}^{3}$ ( $\alpha$ and $\beta$ are constants) is a nonlinear function in $\dot{\varphi}_{r}$ determined experimentally from the standard spiral test. The original nonlinear system can be piecewisely linearized under some cruise conditions. In this research, the nominal cruise speed is set to be 20 knots. The nominal rudder angle, heading angle and the change rate of heading angle are set to be zero degree in this simulation. Furthermore, for a more complete description of the ship model, the rudder servomechanism has to be taken into account. For instance, a natural constraint of the steering machine is given by

$$
-\delta_{\max } \leq \delta \leq \delta_{\max }
$$

where $\delta_{\max }=35$ (degree) is the maximum rudder angle.

In general, the environmental disturbance of the ship steering should be considered, especially the wave, which can affect the yaw angle of ship heading. For simplicity of illustration, the disturbance $f(t)$ in (1b) is generated by

$$
f(s)=h(s) \omega(s),
$$

where $\omega(\bullet)$ is a zero-mean Gaussian white noise and the transfer function $h(s)$ is given by

$$
h(s)=\frac{K_{\omega} s}{s^{2}+2 \zeta \omega_{0} s+\omega_{0}^{2}} .
$$

In (19), $\zeta$ is the damping coefficient, $\omega_{0}$ is the dominating wave frequency, and $K_{\omega}=2 \xi \omega_{0} \delta_{\omega}$ is the

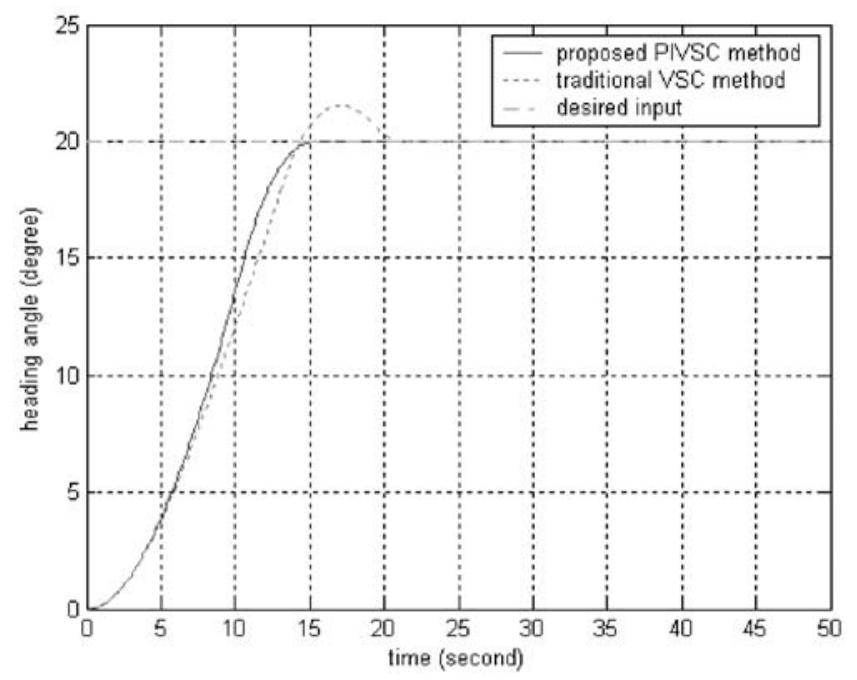

Fig. 2. Simulation results for set point control. gain constant with $\delta_{\omega}$ representing the wave intensity [6]. We then take $F(t)$ in (3) as $F(t)=|f(t)|$.

The conventional maneuvering problems can be classified into four types, namely course keeping handling, course changing handling, course tracking handling, and special motion handling (for instance, marine search handling, rescue handling). In this section, the handling problems of course keeping, course changing, and course tracking, will be investigated by using the proposed PI controller. The related parameter are given as $N_{a i}=0.0206, N_{b}=0.0022, K=0.093, T=$ $8.7, \alpha=9.42, \beta=2.24, \omega_{0}=1.0, \zeta=0.1, \delta_{\omega}^{2}=10, \omega_{1}=$ $0.188, K_{P}=10, \mathrm{c}_{1}=K_{P}+w_{1}$, and $K_{I}=15$. The proportional-gain $K_{P}$ and integral-gain $K_{I}$ are chosen by the designer. In our experiment, the larger the proportional-gain will produce slower response but smaller over-shoot, whereas the larger integral-gain will produce faster response but larger over-shoot.

In the first simulation, the course keeping problem is considered. The main goal of course keeping handling is to keep the heading angle of vessel on the fixed direction. The desired output is set to be twenty degree. The output responses by using the proposed controller (solid line) and by using traditional variable structure controller (dotted line) are shown in Figure 2. From Figure 2, the rise time and overshoot of the output response by using the proposed controller is reasonably well as compared with that by using the traditional variable structure controller.

The course changing handling usually occurs in the collision avoidance problems. For course changing, autopilot control may require large heading angle change and the heading angle of the vessel must turn to the desired direction as soon as possible. In the next simulation of course changing, the vessel is required to

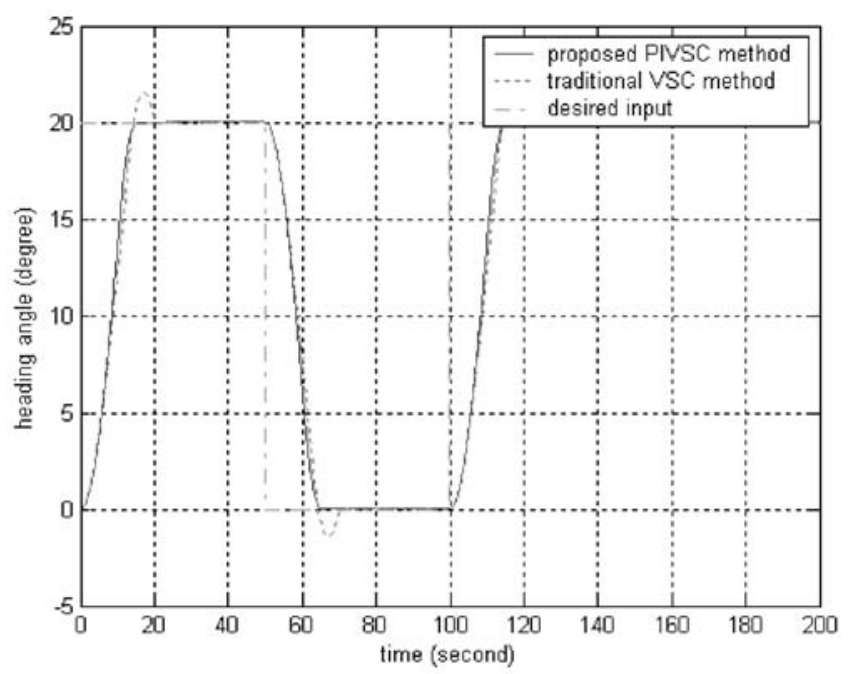

Fig. 3. Simulation results for command change control. 


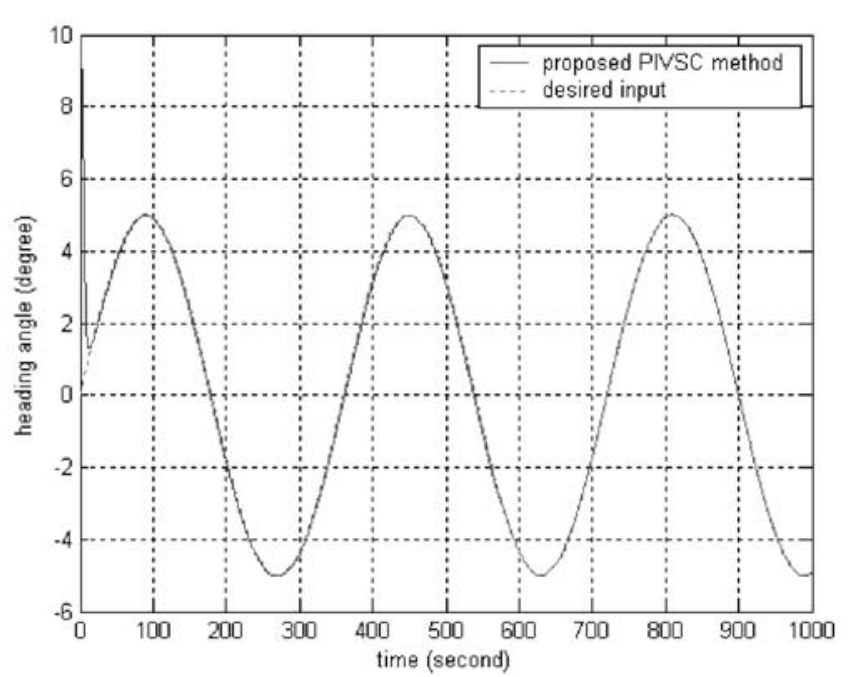

Fig. 4. Simulation results for tracking control.

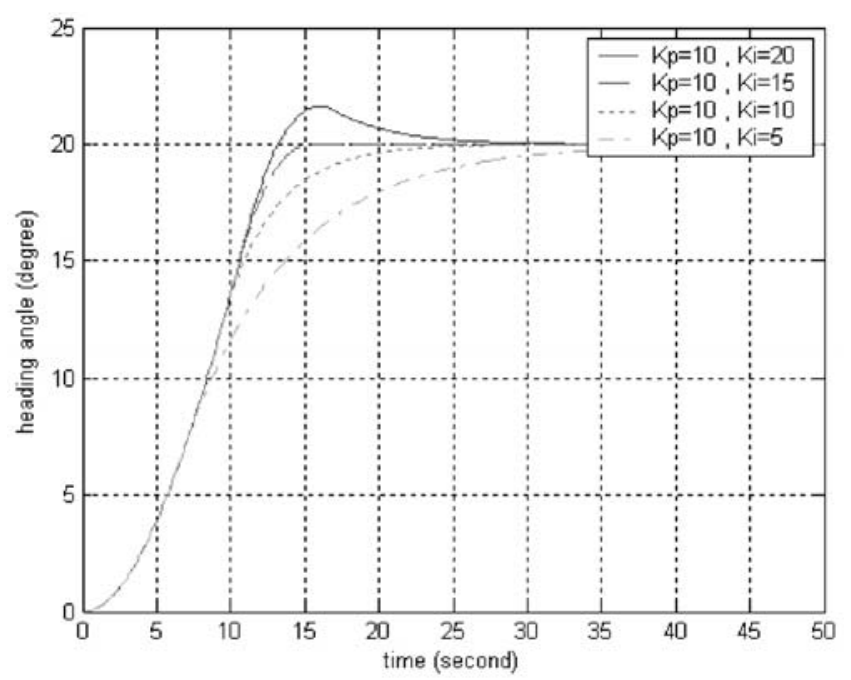

Fig. 5. Simulation results for fixed $K_{P}$ but varied $K_{I}$.

track a sequence of step functions of heights 20,0 , and 20 , respectively, in every 50 second. The simulation results are shown in Figure 3. It is apparent that the performance of the proposed controller is quite good.

The problem of ship maneuvering in restricted water, such as harbors, canals, river inlets, etc., is of major concern from the viewpoint of marine safety. It is very important for a shipmaster to make a shiphandling plan before approaching such maritime space just mentioned. The vessel must navigate in the desired course precisely for safety. This is one form of the course tracking problems. In the final simulation of course tracking handling, the desired heading angle $\varphi_{d}$ is $5 \sin (0.1 \pi t / 180)$. The simulation results are shown in

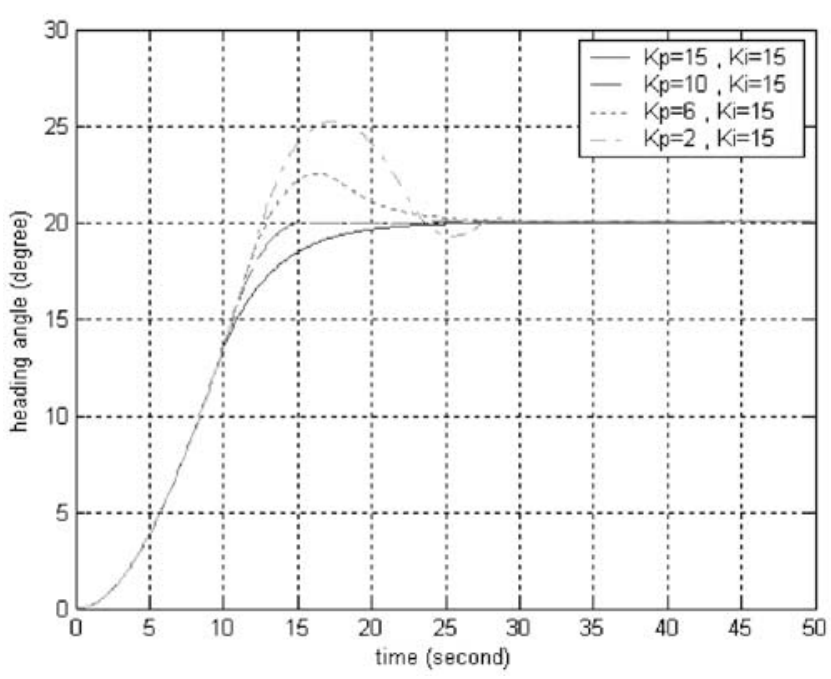

Fig. 6. Simulation results for fixed $K_{I}$ but varied $K_{P}$.

Figure 4. It is obvious that the desired angle and the actual angle responses are almost identical.

The choice of parameters $K_{P}$ and $K_{I}$ is based solely upon our experience. Figure 5 shows the simulation results for fixed $K_{P}$ but varied $K_{I}$ and Figure 6 shows the simulation results for fixed $K_{I}$ but varied $K_{P}$. We wish to point out that some global optimizer, e.g., genetic algorithm, may help determining better $K_{P}$ and $K_{I}$. This constitutes an interesting future research topic.

\section{CONCLUSION}

In this paper, the marine vessel maneuvering problems have been solved by using the variable structure control with proportional and integral controllers. Several ship handling simulations have been made to illustrate our main ideas. We firmly believe that the proposed controller is useful not only for the course keeping problems, course changing problems, and course tracking problems, but also for the maritime search problems and rescue problems.

\section{ACKNOWLEDGMENTS}

This work was supported by the National Science Council, Taiwan, Republic of China, under Grant NSC 92-2611-E-022-005.

\section{REFERENCES}

1. Åström, K.J. and Wittenmark, B., Adaptive Control, Addison-Wesley, Massachusetts (1989).

2. Chen, Y.P. and Her, W.C., "Bang-Bang Controller Design for Servomechanisms Using the Sliding-Mode 
Theory," Proceedings of International Conference on Intelligent Control and Instrumentation, Singapore, 1, 312-316 (1992).

3. Cheng, C.C. and Liu, I.M., "Design of MIMO Integral Variable Structure Controllers," J. Franklin Inst., Vol. 336, pp. 1119-1134 (1999).

4. Chern, T.L. and Wu, Y.C., "Integral Variable Structure Control Approach for Robot Manipulators," IEE Proc.$D$, Vol. 139, No. 2, pp. 161-166 (1992).

5. Chiang, H.K. and Tseng, C.H., Design and Implementation of a Grey Sliding Mode Controller for Synchronous Reluctance Motor Drive, Control Engin. Pract., Vol. 12, No. 2, pp. 155-163 (2004).

6. Fossen, T.I., Guidance and Control of Ocean Vehicles, John Wiley and Sons, New York (1994).

7. Fung, R.F., Wang, Y.C., Yang, R.T., and Huang, H.H., "A Variable Structure Control with Proportion and Integral Compensations for Electro-Hydraulic Position Servo control System," Mechatronics, Vol. 79, No. 1, pp. 67-81 (1997).

8. Ohtsu, K., Shoji, K., and Okazaki, T., "Minimum-Time Maneuvering of a Ship with Wind Disturbances," Control Engin. Pract., Vol. 4, No. 3, pp. 385-392 (1996).

9. Rurns, R. and Richter, R., "A Neural-Network Approach to the Control of Surface Ships," Control Engin. Pract., Vol. 4, No. 3, pp. 411-416 (1996).

10. Sam, Y. Md., Osman, J.H.S., and Ghani, M.R.A., “A Class of Proportional-Integral Sliding Mode Control with Application to Active Suspension System," System Control Lett., Vol. 51, No. 3-4, pp. 217-223 (2004).

11. Slotine, J.J.E. and Li, W., Applied Nonlinear Control, Prentice-Hall, New Jersey (1991).

12. Sutton, R. and Towill, D.R., "Fuzzy Logic Models of the Control Behavior of a Helmsman Performing Coursekeeping and Course-changing Tasks," Proceeding of $8^{\text {th }}$ Symposium on Ship Control Systems, Bath, U.K., 1.1011.119 (1987).

13. Tu, Y., "Automatic Scheduling and Control of a Ship Web Welding Assembly Line," Computers Ind., Vol. 29, pp. 169-177 (1996).

14. Utkin, V.I., "Variable Structure Systems with Sliding Modes," IEEE Trans. Automatic Control, Vol. 22, No. 2, pp. 212-222 (1977).

15. Yau, H.T., "Design of Adaptive Sliding Mode Controller for Chaos Synchronization with Uncertainties," Chaos, Solitons Fractals, Vol. 22, No. 2, pp. 341-347 (2004).

16. Yavin, Y., Frangos, C., and Miloh, T., "Computation of Feasible Control Trajectories for the Navigation of a Ship around an Obstacle in the Presence of Sea Current, " Mathemat. Computer Model., Vol. 21, No. 3, pp. 99117 (1995).

17. Yu, K.W., Hwang, R.C., and Hsieh, J.G., "Automatic Ship Handling of Maritime Search Mission by Using Self-tuning Fuzzy Gain Scheduling PD Controller," J. Navigat., Vol. 52, No. 3, pp. 378-387 (1999). 